\title{
Pion-pion scattering with physical quark masses
}

\section{Daniel Hoying*}

Brookhaven National Laboratory, 20 Pennsylvania St., Upton, NY, 11973;

University of Connecticut, 2152 Hillside Road, unit 3046, Storrs, CT, 06269

E-mail: daniel.hoying@uconn.edu

We present preliminary results on the scattering of pseudoscalar, vector, and scalar mesons on a physical pion mass, $2+1$ flavor mobius-DWF, ensemble with periodic boundary conditions (PBCs) generated by the RBC and UKQCD collaborations. Using all-to-all propagators, we produce thousands of correlator momentum combinations. Energy spectra and phase shifts, including excited states, are then extracted via the solutions of a generalized eigenvalue problem. Included in this talk will also be an overview of the computational strategies employed, including a discussion of split-CG matrix solvers (communication avoidance) and lattice crossing symmetry (momentum combinatorics reduction). These studies are intended to serve as groundwork for a full PBC calculation of direct $\mathrm{CP}$ violation in $\mathrm{K}$->pipi later this year.

The 36th Annual International Symposium on Lattice Field Theory - LATTICE2018

22-28 July, 2018

Michigan State University, East Lansing, Michigan, USA.

\footnotetext{
* Speaker.
} 


\section{Correlator Production}

We use all-to-all propagators[?] (with $n_{\text {hits }}$ set of random sources) to calculate two-point functions. We invert using a Zmobius (non-unitary) domain wall kernel on 185 configurations AMA corrected with 7 configurations using a Mobius (unitary) kernel. These corrections have a very small effect, most likely due to the $1 e-6$ Zmobius stopping condition. We create and annhiliate pions at an offset of 3 time slices to give smaller overlap with the vacuum. We refer to the source pions which are closest to the sink (and vice-versa) in the forward time direction as inner pions. $t_{d i s}$ is the source sink separation between inner pions. We set a maximum $t_{d i s}$ to save on compute time. For the connected diagrams, we also only measure a source on every 8th time slice (the disconnected diagrams dominate the noise so are measured on every time slice).

\section{Production Parameters}

\section{GEVP, Operator Basis}

We first form a matrix of two-point correlators whose dimensions are defined by the same operator basis at source and sink. These operators are $\pi \pi$ operators with different energy overlaps.

For $I=0,2$ we project onto the $A_{1}$ irrep. For $I=1$ we project onto the $T_{1}^{-}$irrep. We include operators with gamma structure $\bar{\psi} \gamma^{5} \psi, \bar{\psi} \gamma^{\mu} \psi, \bar{\psi} \psi$ which we refer to as the pion $(\pi), \rho$, and $\sigma$ operators respectively. Allowed individual 3-momenta of these operators goes up to $\pm(1,1,1)$ ( \pm all permutations of the magnitudes and signs are used in the projection: e.g. $(1,-1,0),(0,-1,0)$, etc.). We average over irrep rows and source time slice. We solve a generalized eigenvalue problem (GEVP) for each separate isospin and center of mass momentum.

$$
\begin{aligned}
C(t) v\left(t, t_{0}\right) & =\lambda\left(t, t_{0}\right) C\left(t_{0}\right) v\left(t, t_{0}\right) \\
E & =-\frac{1}{\left(t-t_{0}\right)} \log \left(\lambda\left(t, t_{0}\right)\right)
\end{aligned}
$$

We include the backwards propagating contribution in our model for $\lambda$, but in practice this is usually negligible since it is suppressed by $e^{-E L_{t}}$.

For $I=2$, we fix $t_{0}=t-1$ since this channel is not very noisy. For $I=0$ we fix $t_{0}=\left\lceil\frac{t}{2}\right\rceil$ as this is the earliest in time (and hence least noisy) time slice we can select which still gives us the best known systematic asymptotic error bound[?] on our energies (which are extracted as $E$ in eq. (3.2))

We calculate all elements of the GEVP matrix and enforce hermiticity by setting $C(t) \rightarrow$ $\frac{1}{2}\left(C(t)+C^{\dagger}(t)\right)$. If we encounter imaginary eigenvalues (usually found at late times), we exclude these time slices. If we encounter negative eigenvalues (which would not a give real log), we omit these energies from our fits (but we may plot the remaining real energies). We do not truncate the operator basis if we are doing a fit (although this can possibly allow us to extract some late time information which would otherwise be inaccessible due to inherent numerical instability - see section 9). 
Table 1: Production Parameters

\begin{tabular}{|c|c|}
\hline Parameter & Value \\
\hline Lattice size & $24^{3} \times 64 \times 12$ \\
\hline coarse grid block size & $12 \times 2 \times 3 \times 3 \times 2$ \\
\hline Spatial Boundary Conditions & Periodic \\
\hline Time Boundary Conditions & Anti-periodic \\
\hline $\mathrm{nl}$ & 2000 \\
\hline nl, fine grid & 1000 \\
\hline nhits & 1 \\
\hline tsep & 3 \\
\hline tstep & 8 \\
\hline tdis max & 16 \\
\hline pion radius & 1.5 \\
\hline rho radius & 1.5 \\
\hline sigma radius & 1.5 \\
\hline$L_{t}$ & 64 \\
\hline beta & 1.633 \\
\hline Ls (Zmobius approx of Mobius Ls=24, Shamir Ls=96) & 12 \\
\hline Ms & 0.0850 \\
\hline $\mathrm{Mu}$ & $1.07 \mathrm{e}-03$ \\
\hline \# Configurations (sloppy only, no MADWF) & 66 \\
\hline $\mathrm{Nh}$ & 768 \\
\hline Random num type & $\mathrm{U}(1)$ \\
\hline $\mathrm{M}_{5}$ & 1.80 \\
\hline Gauge & Iwasaki+DSDR \\
\hline Gauge Fix & Coulomb \\
\hline Gauge Fix residual & $1 \mathrm{e}-14$ (see eq. 8 in [?]) \\
\hline Lanczos (true) rsd & $1 \mathrm{e}-06$ \\
\hline CG stop rsd & $1 \mathrm{e}-08$ \\
\hline CG method & Single-prec, 400 iterations \\
\hline Avg. CG True Resid & $1 \mathrm{e}-06$ \\
\hline RNG Seed & (fixed to config number) \\
\hline
\end{tabular}

\section{Lattice Spacing Corrections}

We noticed significant discrepancies between the continuum dispersion relation $\sqrt{m^{2}+p^{2}}$ and the fits to single pion correlators on this ensemble. Thus, it was necessary to provide a way to correct our $E_{\pi \pi}$. We chose to add a correction defined by

$$
E_{\pi 1}+E_{\pi 2}-\sqrt{m_{\pi}^{2}+p_{1}^{2}}-\sqrt{m_{\pi}^{2}+p_{2}^{2}}
$$

where $p_{j}=\frac{2 \pi n_{j}}{L}$ are lattice momenta and $E_{\pi j}$ is a fit to a $\pi$ constructed with this momentum. For $I=0,1$ channels, the presence of the $\sigma, \rho$ shift the energies away from their non-interacting 
values (giving them quite large phase shifts). For these channels, an alternative dispersion relation (guessed from naive scalar field theory) is used which gives good agreement with fits to $E_{\pi}$ :

$$
\begin{aligned}
E_{\mathrm{disp}} & =\sqrt{m^{2}+\hat{p}^{2}} \\
\hat{p} & \equiv 2 \sin \left(\frac{n \pi}{L}\right)
\end{aligned}
$$

\section{Other processing}

For $I=0$, we do a vacuum subtraction defined due to the existence of disconnected diagrams.

$$
\left\langle O_{s n k} O_{s r c}^{\dagger}\right\rangle_{s u b} \equiv\left\langle O_{s n k}(t) O_{s r c}^{\dagger}(0)\right\rangle-\left\langle O(t)_{s n k}\right\rangle\left\langle O_{s r c}^{\dagger}\right\rangle
$$

We know we have around the world contributions to our energy eigenvalues. For moving frames, these are also dependent on time separation. Each subtraction is defined by $C(t)-C\left(t-\delta_{t}\right)$ with approriate weighting for the time dependence (see [?] for a much more detailed explanation). We subtract up to and including second order in around the world contribution. We use a time difference $\delta_{t}=3$ for each subtraction which is chosen to be the empirical minimum which has the best noise properties. To be more explicit, if we start with $\delta_{t}=1$ or $\delta_{t}=2$ we can still significantly reduce noise by going to $\delta_{t}=3$. Larger $\delta_{t}$ do not seem to do much to the noise levels. We did not check this for every GEVP solve, but we did test it enough that it seems to be invariant under a wide variety of inputs. It is an open problem to provide an explanation for this behavior.

Each time we perform a subtraction, we use $\delta_{t}$ time slices, so $t$ shown often starts at 4 or 7 depending on if we are looking at the center of mass frame or some boosted frame.

Due to $I=1$ providing asymmetry to the wave function, no around the world subtraction is necessary for the center of mass frame.

\section{Fitting}

We fit under a jackknife the processed energy values simultaneously to $N$ constants for an $N x N$ GEVP. It can be somewhat difficult to find a good subset of time slices to fit to, so we loop over subsets pseudo-randomly (to avoid bias) until we get a decent fit. We then extend the time slice inclusions as far as we can dimension by dimension (to minimize overfitting) until we get a p-value for our $\chi^{2} / d o f$ which is $>0.1$ (our arbitrarily chosen cutoff). Some fits clearly lack a very stable plateau and we expect that fitting to a constant underestimates excited state contamination especially in the higher energy states of $I=0,1$. Even for $I=2$, there are likely significant possible improvements once we estimate this contamination (future work). For now, we estimate by eye when we have a stable plateau. We use this estimation to set our $t_{\min }$. For $t_{\max }$, we exclude from our fits any point which relative error $>20 \%$ (or where the GEVP has what appears to be numerical instability causing sudden jumps in the energies and very large error bars on one or more states). The points with large error bars do not do much to constrain the fit value and cause spurious contributions of $\sim 1$ to $\chi^{2} / d o f$.

Scattering phase shifts are extracted via the Lüscher method via code derived from [?]. 


\section{7. $I=2$ (Preliminary)}

We plot here all the various center of mass momenta as well as a phenomenology (from Roy equations) and chpt curves. The points discrepant might be due to unsubtracted around the world contributions or lattice spacing errors. Excited state contamination is expected to lower the phase shifts, so this effect must not be too large. Moreover different fit ranges give non-compatible results (as expected if there was contamination). Work is ongoing to try to understand this dependence (and the corresponding discrepancies with phenomenology). However, the ground states of the various moving frames exhibit discrepancies which are fairly independent of fitted time slices (and so may share a common origin).

\subsection{Phase Shift vs. $\sqrt{s}$}

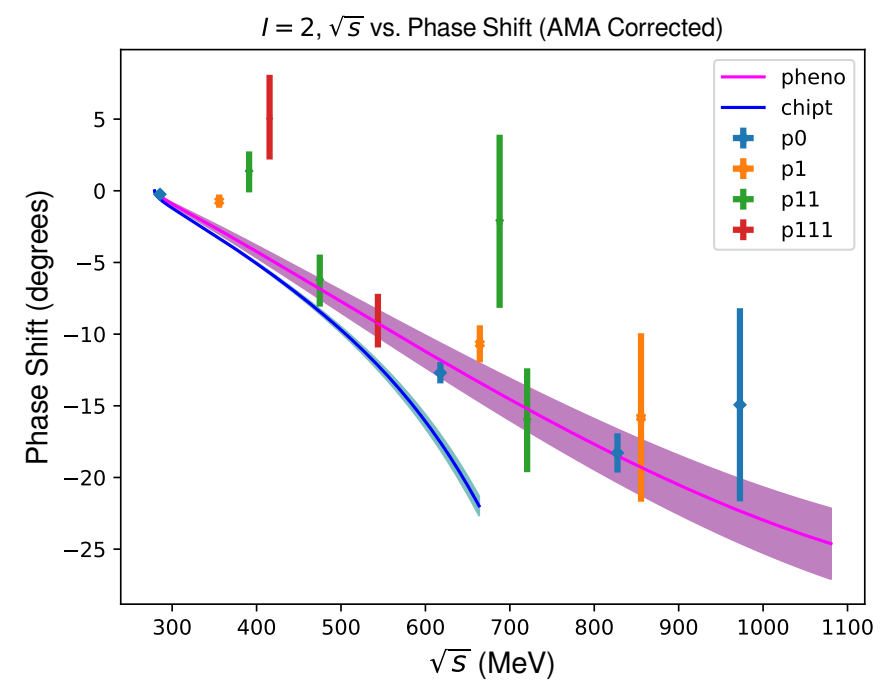

\subsection{Representative Plot}

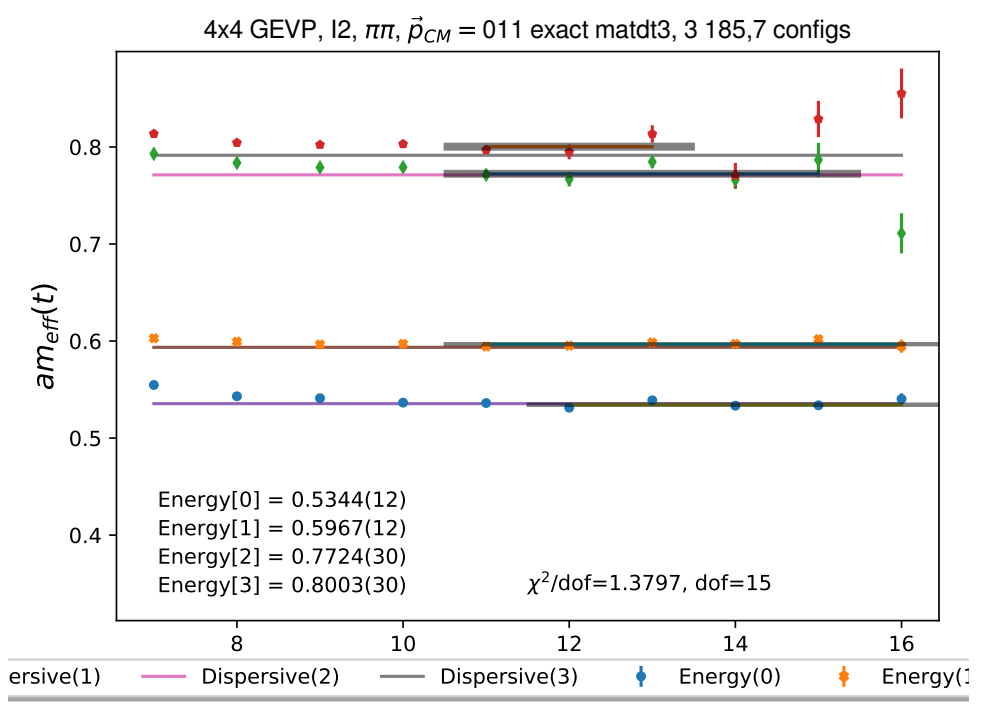




\section{8. $I=0$ (Preliminary)}

\subsection{Representative Plot}

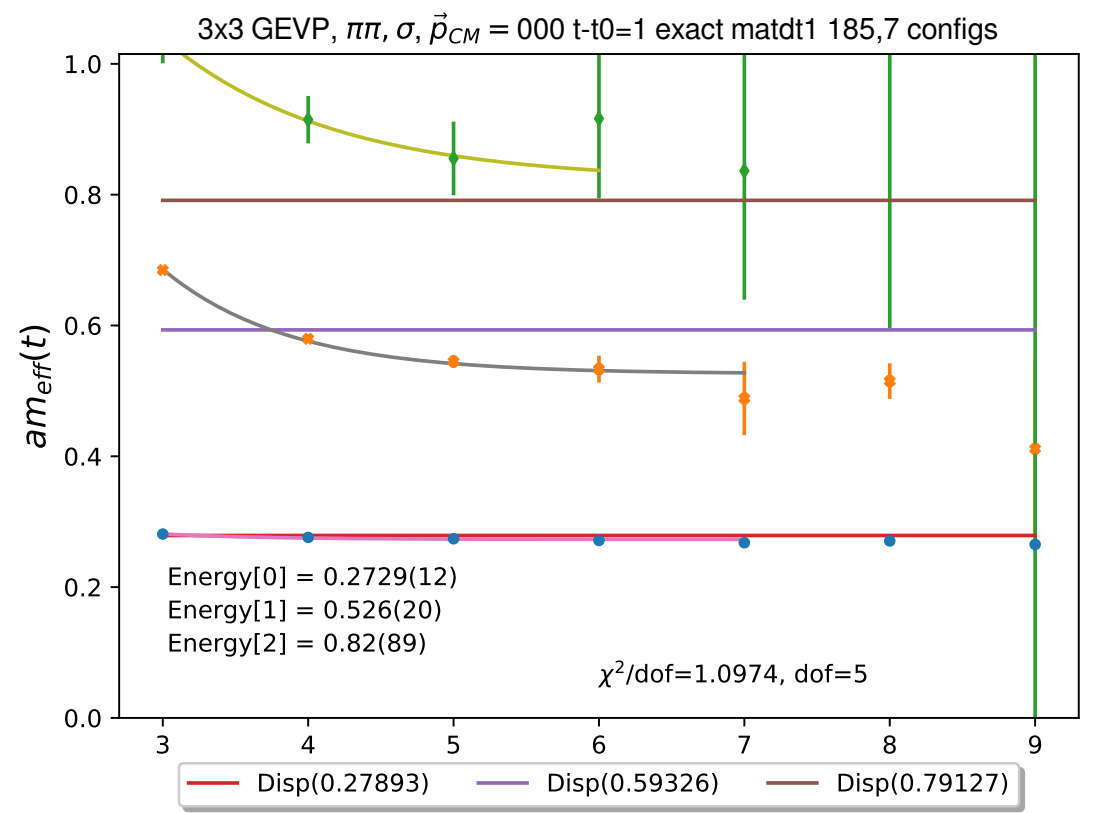

Figure 1: p0

\begin{tabular}{c|c}
$\sqrt{s}(\mathrm{MeV})$ & Phase Shift (degrees) \\
$277.0(1.2)$ & N/A \\
$534(21)$ & $48(13)$ \\
$833(908)$ & $-19(66)$
\end{tabular}

Table 2: p0 Numerical Values, the ground state is below $2 m_{\pi}$ in energy, so the phase shift is complex (and omitted). We estimate excited state contamination for $E_{n}$ by adding to our constant fit an extra exponential: $a m_{e f f, n}=E_{n}+a_{n} e^{-\left(E_{N+1}-E_{n}\right) t}$. We fix $t-t_{0}=1$. For the $\pi \pi$ operators, we also add (from dispersion relation) and subtract (from single $\pi$ correlator squared) the free $2 \pi$ energy in order to reduce noise.

\section{9. $I=1$ (Preliminary)}

N.B. We have fewer configurations for $I=1$ : 155 with 7 used for the AMA correction. The analysis is still in progress here, so we only show a non-fitted plot for illustration.

Also, we are employing two techniques to reduce the noise not used in the other isospins. The first, as mentioned in section 3 is simply to delete operators at late times starting with the highest $\pi \pi$ energy ( $\rho$ is the second to last to be eliminated). We also notice eigenvalues may switch order from jackknife sample to jackknife sample (even if we sort them by size). This order switch can be detected by checking the ordering to the corresponding eigenvectors (which are usually more stable). We can create an identification of the eigenvectors (even over jackknife samples) by 
maximizing inner products with the eigenvectors found from the average correlator matrix $\langle C(t)\rangle$ (over jackknife samples).

However, due to time constraints, we do not have code to do this yet so we employ a slightly older technique which has for $\varepsilon \ll 1$ (set to be as small as possible without causing precision loss)

$$
\begin{array}{r}
C(t) \rightarrow(C(t)-\langle C(t)\rangle) \varepsilon+\langle C(t)\rangle \\
E \rightarrow(E-\langle E\rangle) \frac{1}{\varepsilon}+\langle E\rangle
\end{array}
$$

This introduces bias on the order of the jackknife bias (one can show) so can be considered safe in regions where the error is small enough that the jackknife bias can be neglected. This technique should give consistent results with the eigenvector identification method, but this has not yet been checked. As this is mostly for illustration purposes, we assume that the errors are small enough to make the technique reliable.

\subsection{Representative Plot}

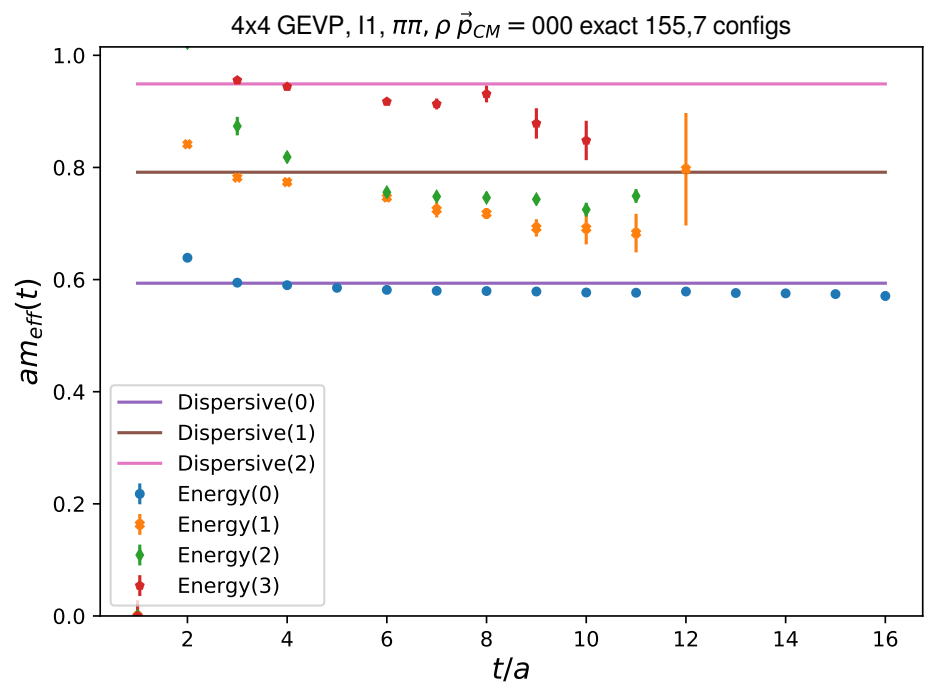

